\title{
Clinical Course and Outcome of 395 Covid 19 Patients Admitted to One Hospital in Jeddah- Saudi Arabia
}

\author{
Samar Assem Badreddine $e^{1, *}$, Mohammed Zammo ${ }^{1}$, Abdullah Abdelfattah Elhosiny ${ }^{1}$, \\ Mohanna Walid Alhomsy ${ }^{1}$, Yasser Aldabbagh ${ }^{1}$, Abdullah Sameer Mansouri ${ }^{1}$, Sara Hesham Taha ${ }^{1}$, \\ Reem Yahya ALQuraa ${ }^{1}$, Abdulaziz Abdullah Al Nahdi ${ }^{1}$, Ayman Eissa ${ }^{1}$, Hanan Mohammed \\ Faruqui $^{1}$, Nehal Ahmed ${ }^{2}$, Ahmad Alzahrani ${ }^{3}$, Nezar Bahabri ${ }^{1}$ \\ ${ }^{1}$ Department of Internal Medicine, Doctor Soliman Fakeeh Hospital, Jeddah, Saudi Arabia \\ ${ }^{2}$ Department of Infection Control, Doctor Soliman Fakeeh Hospital, Jeddah, Saudi Arabia \\ ${ }^{3}$ Department of Laboratory Medicine, Doctor Soliman Fakeeh Hospital, Jeddah, Saudi Arabia
}

Email address:

sbadreddine@fakeeh.care (S. A. Badreddine)

${ }^{*}$ Corresponding author

\section{To cite this article:}

Samar Assem Badreddine, Mohammed Zammo, Abdullah Abdelfattah Elhosiny, Mohanna Walid Alhomsy, Yasser Aldabbagh, Abdullah Sameer Mansouri, Sara Hesham Taha, Reem Yahya ALQuraa, Abdulaziz Abdullah Al Nahdi, Ayman Eissa, Hanan Mohammed Faruqui, Nehal Ahmed, Ahmad Alzahrani, Nezar Bahabri. Clinical Course and Outcome of 395 Covid 19 Patients Admitted to One Hospital in Jeddah- Saudi Arabia. International Journal of Infectious Diseases and Therapy. Vol. 5, No. 4, 2020, pp. 118-126. doi: $10.11648 /$ j.ijidt.20200504.13

Received: September 17, 2020; Accepted: October 9, 2020; Published: October 17, 2020

\begin{abstract}
Background: Since the first case of SARS-CoV 2 has been reported from Wuhan China back in December 2019, the virus has spread all over the world and has so far infected more than 35 million humans and led to more than one million deaths woldwide. We describe in this paper the clinical characteristics and outcome of PCR confirmed Covid 19 patients that were admitted to a tertiary care hospital in Saudi Arabia. Methods: Retrospective review of patients that had positive PCR on nasopharyngeal swab for SARS Cov2 and that were admitted and discharged from a tertiary care hospital in the city of Jeddah, Saudi Arabia between March and July 2020. Epidemiological, demographic, and clinical data were collected. The clinical course of patients was reviewed. Risk factors for involvement of lower respiratory tract (Pneumonia), for need for ICU and for death were analyzed. Results: The records of total of 395 patients were reviewed. $15 \%$ of Covid 19 patients in our population were completely asymptomatic, one quarter of which had abnormalities on chest imaging. Among the patients with one or more Covid 19 symptoms, $75 \%$ had lower respiratory tract involvement and one quarter had normal chest imaging. One third of all patients developed leukopenia and around 2 thirds had lymphocytopenia. Thrombocytopenia was not common (occurred in $15 \%$ ), $29 \%$ of our patients had CRP $>10$ and $25.1 \%$ had elevated ALT (not exceeding 5 times upper normal). Nine percent of our patients needed ICU admission, 3.8\% needed mechanical ventilation. 9 patients $(2.3 \%)$ in our population died. Advancing age, increasing BMI, and smoking history were significantly associated with increased mortality. Developing abnormalities on chest imaging (Pneumonia) was significantly associated with increasing BMI, advancing age, not receiving BCG vaccination at birth, history of smoking and presence of co-morbidities ( $\mathrm{p}$ value less 0.05 with all these variables). Blood group and presence of co-morbidities was significantly associated with need for ICU care but not with mortality. In our population neither ethnicity, nor gender, had significant association with hospital course or outcome, and no one younger than 45 years and no one with BMI less than 24 died. Conclusion: Advancing age, increasing BMI and history of smoking were found to be significant risk factors for mortality in our population. History of Bacille calmette Guerin (BCG) vaccination was significantly associated with less involvement of lower respiratory tract but had no significant association with final outcome. Asymptomatic Covid 19 is more of a silent active infection rather than a silent inactive carrier state.
\end{abstract}

Keywords: Covid 19, Saudi Arabia, BCG Vaccine 


\section{Introduction}

SARS- Cov-2, is an RNA novel coronavirus that was first reported in Wuhan China in December 2019 [1]. Since then the virus has spread to all countries on planet earth and has been reported from almost every country. The virus causes a spectrum of illness ranging from asymptomatic illness, to upper respiratory tract infection, lower respiratory tract infection and severe ARDS like picture.

At the time of writing this paper, the total number of cases reported by WHO worldwide has exceeded 35 million, and mortality from the virus has exceeded one million deaths. Saudi Arabia was no exception and Covid 19 was reported first in Saudi Arabia on March $2^{\text {nd }}, 2020$ [2]. Till the time of writing this paper Saudi health officials have reported total of 337000 Infections and 4898 deaths from the virus.

Infection with SARS Co-v2 virus has been reported to cause asymptomatic disease in up to half of those that are infected [3]. In symptomatic patients, the virus causes a spectrum of clinical presentations that includes fever, cough, shortness of breath, myalgias, sore throat $[4,5]$, diarrhea and other gastrointestinal symptoms [6] The virus can involve both upper and lower respiratory tracts, with the latter being more common. Pneumonia can progress into acute respiratory distress syndrome like picture (ARDS) and can in a small minority lead to death. The severity of the disease seems to be associated with certain demographic characteristics of patients and with presence of comorbidities [7]. Lymphocytopenia [8] and presence of bilateral peripheral ground glass opacities on Computed tomography of chest [9] have been reported to be amongst the most common findings on diagnostic work up of covid 19 patients.

We are reporting in this paper the experience of a tertiary care center in Saudi Arabia with Covid 19 patients. We are reviewing the epidemiologic and clinical characteristics of patients that tested positive on nasopharyngeal PCR for SARS-Cov 2 and that were admitted to regular wards of our hospital between March and July 2020. We are also describing the epidemiologic and clinical characteristics, the hospital course of those patients and their outcome.

\section{Methods}

Dr. Soliman Fakeeh hospital is a tertiary care hospital located in the city of Jeddah, Saudi Arabia. This city has a population of around 4 million people and was affected by the Covid 19 pandemic like all other cities in Saudi Arabia. Our hospital is one of the admissions destinations in the city for management of Covid 19 patients.

The Hospital Admission criteria of Covid 19 patients in Saudi Arabia are based and are in compliance with the guidelines / recommendations of the Saudi ministry of health $(\mathrm{MOH})$. Early in the pandemic (March - April) hospitals were mandated to admit all suspected (meet Saudi Arabia $\mathrm{MOH}$ case definition of suspected Covid 19) or confirmed
Covid 19 patients (patients with positive nasopharyngeal PCR for SARS Cov-2) regardless of whether patients were symptomatic or not. Hospital Admission criteria changed later in May and excluded Asymptomatic patients and included only symptomatic patients with or without evidence of pneumonia, desaturation, or comorbidities. These criteria then changed again in June 2020 to limit admission only for patients with evidence of pneumonia and desaturation. Admitted patients to our hospital were managed during hospital stay based on management protocols set by the infectious diseases section at Dr. Soliman Fakeeh hospital. These protocols were derived from most current published scientific evidence and guidelines and were aligned with Saudi MOH Covid 19 management guidelines. Accordingly, our Covid 19 management protocols were amended multiple times since March and changes were made based on evolving scientific findings. For example, hydroxychloroquine and / or azithromycin were initially standard practice to all symptomatic confirmed patients, later we dropped combination therapy and stopped azithromycin and afterwards dropped Hydroxychloroquine (concern on cardiac toxicity - prolonged QI interval). We started using Tocilizumab in patients with desaturation towards the end of May, and then started giving dexamethasone for patients with pneumonia and in need of supplemental oxygen after the RECOVERY study results were published. Since the beginning It was our standard (and continues to be) to give prophylactic anticoagulation to all asymptomatic or mildly symptomatic patients (with upper respiratory tract infection and no evidence of pneumonia) and to give full anticoagulation to patients whose duration of fever exceeded 5 days or to those with evidence of pneumonia on chest imaging.

In this paper we included all patients with confirmed Covid 19 (Positive nasopharyngeal PCR for SARS Cov-2) that were admitted to regular wards in our hospital between March - July 2020. We excluded Covid 19 patients that presented with severe respiratory distress necessitating direct admission to critical care unit.

\section{Result}

We reviewed the records of 396 Adult patients who had positive SARS-CoV 2 PCR on nasopharyngeal swabs and that were admitted to regular wards of our hospital between March and July 2020. We excluded patients that met admission criteria to critical care units.

Epidemiologic characteristics (Table 1): Majority of patients in our population (51.6\%) were aged between 18 and 45 years, $36.7 \%$ were between age 45 and 65 and $9.4 \%$ were older than 65 years. $70.4 \%$ were male, and $29.6 \%$ were females. Half of patients were Caucasian $50.6 \%, 41.8 \%$ were of Asian origin and $7.6 \%$ of African origin. $41.8 \%$ were Saudi nationals.

Of those for whom BMI was captured (239 patients), 48\% had BMI between 24-30, 32\% had BMI between 30-40, and 


\section{$5 \%$ had $\mathrm{BMI}>40$.}

Of those for whom smoking history was obtained (296 patients), 74\% were nonsmokers and of those from whom we obtained history of BCG vaccination (237 patients), $80.5 \%$ received the vaccine directly after birth.

Blood group was obtained for 221 patients, 35.3\% were $\mathrm{O}+$, followed by $\mathrm{A}+(30 \%)$ then $\mathrm{B}+(23.5 \%)$. Negative $\mathrm{Rh}$ accounted for $5.8 \%$.

Almost half of our patients $(46.6 \%)$ did not have any comorbidities, $23.6 \%$ had diabetes, $19.6 \%$ had hypertension and $4.0 \%$ had cardiac diseases.

Almost half of our patients $(56.2 \%)$ denied any known exposure to Covid 19 patients or history of travel in prior 14 days before onset of symptoms.

Clinical characteristics (Table 2): Total of 59 patients $(15 \%)$ of confirmed Covid 19 patients were completely asymptomatic. Total of 84 patients (21.2\%) were symptomatic and had normal chest imaging suggesting an upper respiratory tract infection, and the rest (252 patients$63.8 \%$ ) developed pneumonia.

Of those with symptoms, history of fever was the most common presenting symptom $(74.2 \%)$, followed by cough $(65.6 \%)$ shortness of breath (40.3\%) and diarrhea (15.2\%). A smaller group had anosmia, dysgeusia and sore throat.

389 patients had $\mathrm{O}_{2}$ saturation documented upon admission. Of these $\mathrm{O}_{2}$ saturation on Room Air was $\geq 94 \%$ in majority of patients $(85 \%)$, was between $90 \%$ and $93 \%$ for $8.9 \%$ of patients and was less than $90 \%$ for $6.1 \%$ of patients.

Laboratory findings (Table 2): 124 patients (31.4\%) had White blood cells less than 5.0 on admission and additional 19 patients who had normal WBC on admission dropped their WBC to less than 5.0 during hospital stay. 13 patients (3.3\%) had WBC on admission greater than 11 (leukocytosis) without evidence of concurrent bacterial process. The highest WBC encountered in our population in patients with clinical illness suggestive of Covid and with no evidence of concurrent bacterial infection was 12.2; the involved patient had normal lymphocyte percentage.

239 patients $(60.5 \%)$ had lymphocyte percentage less than $25 \%$ on admission and additional 23 patients who had normal lymphocyte percentage on admission dropped it to $25 \%$ or less during admission. 14 patients (3.5\%) had lymphocytosis on admission (lymphocyte percentage more than $44 \%$ ).

60 patients $(15.1 \%)$ had thrombocytopenia (platelets less than 150,000). Only 2 patients had platelets less than 80,000 .

Most patients that were tested for liver function tests upon admission (total 323 patients) had ALT $=<40$ (74.9\%). 81 patients $(25.1 \%)$ had ALT $>40,5$ patients had ALT between 100 and 137 and only one patient who had a smooth hospital course and discharged had ALT higher than 200 (ALT 238).

383 patients had CRP tested upon admission. Total of 111 patients (29\%) had CRP greater than 10. Of these, 45 patients (11.7\% of total) had CRP > 100, 23 of which CRP was between 100 and 150 . Only 7 patients had CRP $>200$, and all of them were discharged home in good condition. The highest CRP reported in our series was 261.

D-dimer was done upon admission for 236 patients and was less or equal to 0.5 in 114 patients $(48.3 \%)$. LDH was tested upon admission for 233 patients and was found to be elevated ( $\mathrm{LDH}>225)$ in 191 patients $(82 \%)$

Radiologic findings (Table 2): Total of 392 patients that had Chest X-ray (CxR) done during admission. Of these 177 patients (45.1\%) had normal CxR. The remaining (215 patients) had abnormal CxR with findings consisting in 162 patients $(41.3 \%)$ of bilateral involvement of lungs (with opacities, infiltrates, and consolidation), and of unilateral involvement (infiltrates, consolidations, opacities) in 53 patients $(13.7 \%)$. Total of 155 patients had High resolution CT (HRCT) done, with 120 patients (77.4\%) showing bilateral ground glass opacities, 13 patients $(8.4 \%)$ had Unilateral ground glass opacities and 21 patients $(13.5 \%)$ had normal HRCT.

Total of 266 patients $(67.2 \%)$ had abnormal chest imaging either on Chest X-ray or on high resolution CT of chest (HRCT). In patients with one or more covid 19 symptoms (336 patients), 84 patients (25\%) had normal chest imaging and 252 had abnormal chest imaging (75\%). Of the 66 patients that had normal Chest X-ray and later had HRCT done during same admission, HRCT was abnormal in 49 patients $(74.2 \%)$.

Management (Table 2): Asymptomatic patients did not receive any Covid 19 targeted pharmaceutical therapy. Symptomatic patients and according to which period they were admitted and what recommendations were applicable in our updated management protocol received Covid 19 targeted therapy as follows: (20.6\%) received Hydroxychloroquine (HCQ), (12.5\%) received steroids, (9.9\%) received Tocilizumab, (16.5\%) received Azithromycin, and (17.7\%) received a regimen that contains both azithromycin and Hydroxychloroquine.

Hospital course and outcome (Table 2): 35 patients (8.9\%) of our population were admitted to ICU because of severe respiratory distress and significant desaturation. Of these, 15 $(43 \%)$ needed mechanical ventilation $(3.8 \%$ of total population). Total of 9 patients in our series died, this makes mortality $26 \%$ in those admitted to ICU), $60 \%$ in those that needed mechanical ventilation and overall mortality of our population $2.27 \%$.

Asymptomatic patients: Of the 59 completely asymptomatic patients, $15(25.4 \%)$ had abnormal Chest imaging including Ground Glass Opacities and infiltrates, 2 patients had leukopenia with (WBC less than 5), 25 patients (42.4\%) had lymphocytopenia (lymphocytes percentage less than $25 \%)$ and 8 patients (13.5\%) had elevated LDH (LDH> 225).

Associations and Analysis (Table 3 and table 4): In our chi-square analysis, we found that advancing age had a statistically significant association with development of lower respiratory tract involvement (pneumonia), with admission to ICU, with need for mechanical ventilation and with mortality No one younger than 45 years of age died ( $p$ value 0.013 ).

BMI had a statistically significant association with developing lower respiratory tract involvement (Pneumonia) 
and with outcome: No one with BMI of less than 24 Died. Mortality increased with increase in BMI (p-value 0.043)

Smoking Status had a statistically significant association with developing lower respiratory tract involvement (Pneumonia) and with outcome. 1 (1.3\%) smokers died compared to $2(0.9 \%)$ non-smokers (p-value $<0.014)$.

Blood group had a statistically significant association only with need for ICU admission and need for mechanical ventilation ( $\mathrm{p}-0.000)$ but not with outcome. $\mathrm{O}+$ group being the least likely to need ICU and B+ the most likely to need it.

$B C G$ vaccination had a statistically significant association only with developing lower respiratory tract involvement (Pneumonia, p-value 0.05) but not with hospital course or outcome.

Having co-morbidities showed a statistically significant association with developing lower respiratory tract involvement (Pneumonia, p-value 0.001) and with need for ICU admission ( $p$-value 0.000 ) but not with outcome; Diabetes mellitus and cardiac disease had the highest association.
Our analysis showed that in our Populations neither ethnicity, nationality nor gender had significant association with developing lower respiratory tract involvement nor with hospital course nor with outcome.

Developing abnormalities on chest imaging was significantly associated with increasing BMI, advancing age, not receiving BCG vaccination at birth, history of smoking and with presence of co-morbidities ( $p$ value less 0.05 with all these variables).

Choice of Covid 19 targeted medications showed a statistically significant association with transfer to ICU (pvalue 0.000 ) and with outcome/ mortality (p-value 0.000). In our population those that received Azithromycin had the weakest association with need for ICU admission and with need for mechanical ventilation and those that received Hydroxychloroquine had weakest association with mortality. On the other hand, Tocilizumab had the strongest association with transfer to ICU, need for mechanical ventilation and with mortality.

Table 1. Epidemiologic characteristics.

\begin{tabular}{|c|c|}
\hline Age & $\mathrm{N}=395(\%)$ \\
\hline $18<$ Age $<45$ & $204(51.6)$ \\
\hline $45>$ Age $<65$ & $145(36.7)$ \\
\hline$>65$ & $37(9.4)$ \\
\hline \multicolumn{2}{|l|}{ Gender } \\
\hline Male & $278(70.4)$ \\
\hline Female & $117(29.6)$ \\
\hline \multicolumn{2}{|l|}{ Ethnicity } \\
\hline Caucasians & $200(50.6)$ \\
\hline African & $30(7.6)$ \\
\hline Asian & $165(41.8)$ \\
\hline \multicolumn{2}{|l|}{ Nationality } \\
\hline Saudi Arabian & $165(41.8)$ \\
\hline all others & $230(58.2)$ \\
\hline \multicolumn{2}{|l|}{ BMI $^{*}$} \\
\hline $\mathrm{BMI}<24$ & $34(8.6)$ \\
\hline BMI 24 - 30 & $115(29.1)$ \\
\hline BMI 30-40 & $77(19.5)$ \\
\hline $\mathrm{BMI}>40$ & $13(3.3)$ \\
\hline Not available & $156(39.5)$ \\
\hline \multicolumn{2}{|l|}{ Smoking } \\
\hline Yes & 78 (19.7) \\
\hline No & $218(55.2)$ \\
\hline Not known & $99(25.1)$ \\
\hline \multicolumn{2}{|l|}{$\mathrm{BCG}^{* *}$ vaccine } \\
\hline Yes & $220(55.7)$ \\
\hline No & $53(13.4)$ \\
\hline Not known & $122(30.9)$ \\
\hline \multicolumn{2}{|l|}{ Blood Group } \\
\hline $\mathrm{A}^{+}$ & $66(16.7)$ \\
\hline $\mathrm{B}^{+}$ & $52(13.2)$ \\
\hline $\mathrm{O}+$ & 78 (19.7) \\
\hline $\mathrm{AB}+$ & $12(3.0)$ \\
\hline A- & $4(1.0)$ \\
\hline B- & $2(0.5)$ \\
\hline O- & $7(1.8)$ \\
\hline AB- & 0 \\
\hline Unknown & $174(44.1)$ \\
\hline \multicolumn{2}{|l|}{ Co-morbidities } \\
\hline Diabetes & $118(23.6)$ \\
\hline Hypertension & $98(19.6)$ \\
\hline Cardiac diseases & $20(4.0)$ \\
\hline
\end{tabular}




\begin{tabular}{ll}
\hline Age & $\mathbf{N}=\mathbf{3 9 5}(\mathbf{\%})$ \\
\hline None & $233(46.6)$ \\
Exposure & \\
Contact with Covid-19 patients & $173(43.8)$ \\
NO known contact & $222(56.2)$ \\
\hline
\end{tabular}

*BMI: body mass index.

**BCG: Bacille Calmette Guerin.

Table 2. Clinical characteristics and hospital course.

\begin{tabular}{|c|c|c|}
\hline \multicolumn{3}{|l|}{ 'Symptoms } \\
\hline Fever & & $293(74.2 \%)$ \\
\hline Cough & & $259(65.6 \%)$ \\
\hline Shortness of breath & & $159(40.3 \%)$ \\
\hline Diarrhea & & $60(15.2 \%)$ \\
\hline Asymptomatic & & $59(15 \%)$ \\
\hline \multicolumn{3}{|l|}{ Oxygen $(\mathrm{O} 2)$ saturation on admission $(\mathrm{N}=389)$} \\
\hline equal or more than $94 \%$ & & $330(85 \%)$ \\
\hline between $90-93 \%$ & & $35(8.9)$ \\
\hline less than $90 \%$ & & $24(6.1)$ \\
\hline \multicolumn{3}{|l|}{ White blood cells count (WBC) on admission $(\mathrm{N}=395)$} \\
\hline WBC on admission $>11$ & & $13(3.3 \%)$ \\
\hline WBC on admission $<5.0$ & & $124(31.4 \%)$ \\
\hline \multicolumn{3}{|l|}{ Lymphocytes percentage on admission $(\mathrm{N}=395)$} \\
\hline lymphocyte percentage $<25 \%$ & & $239(60.5 \%)$ \\
\hline lymphocyte percentage $>44 \%$ & & $14(3.5 \%)$ \\
\hline \multicolumn{3}{|l|}{ Platelets count on admission $(\mathrm{N}=395)$} \\
\hline Platelets count $<150,000$ & & $60(50.2 \%)$ \\
\hline \multicolumn{3}{|l|}{ Alanine aminotransferase (ALT) $(\mathrm{N}=323)$} \\
\hline $\mathrm{ALT}=<40$ & & $242(74.9 \%)$ \\
\hline ALT $>40$ & & $81(25.1 \%)$ \\
\hline \multicolumn{3}{|l|}{$\mathrm{C}$ reactive protein $(\mathrm{CRP})$ on admission $(\mathrm{N}=383)$} \\
\hline CRP $1=<10$ & & $272(71 \%)$ \\
\hline $\mathrm{CRP}>10$ & & $111(29 \%)$ \\
\hline \multicolumn{3}{|l|}{ D-dimers on admission $(\mathrm{N}=236)$} \\
\hline $\mathrm{D}$ dimers $=<0.5$ & & $114(48.3 \%)$ \\
\hline D-dimers $>0.5$ & & $122(51.7 \%)$ \\
\hline \multicolumn{3}{|l|}{ Lactate dehydrogenase (LDH) on admission $(\mathrm{N}=233)$} \\
\hline $\mathrm{LDH}=<225$ & & $42(18 \%)$ \\
\hline $\mathrm{LDH}>225$ & & $191(82 \%)$ \\
\hline \multicolumn{3}{|l|}{ Chest X-ray (CxR) findings $(\mathrm{N}=392)$} \\
\hline Normal CxR & & $177(45.1 \%)$ \\
\hline bilateral infiltrates or opacities or consolidation & & $162(41.3 \%)$ \\
\hline unilateral infiltrates or opacities or consolidation & & $53(13.7 \%)$ \\
\hline \multicolumn{3}{|c|}{ High resolutionCcomputed Tomography (HRCT) of chest findings ( $\mathrm{N}=155)$} \\
\hline Normal CT & & $21(13.5 \%)$ \\
\hline bilateral ground glass opacities & & $120(77.4 \%)$ \\
\hline unilateral ground glass opacities & & $13(8.4 \%)$ \\
\hline Others & & $1(0.3 \%)$ \\
\hline \multicolumn{3}{|l|}{ Medications } \\
\hline Supportive care & & $137(17.5)$ \\
\hline Hydroxychloroquine & & $161(20.6)$ \\
\hline Therapeutic anticoagulation & & $170(21.8)$ \\
\hline Steroids & & $98(12.5)$ \\
\hline Tocilizumab & & $77(9.9)$ \\
\hline Azithromycin & & $129(16.5)$ \\
\hline Hydroxychloroquine and azithromycin & & $70(17.7)$ \\
\hline Hydroxychloroquine and Tocilizumab & & $25(6.3)$ \\
\hline \multicolumn{3}{|l|}{ Hospital course } \\
\hline Admission to ICU & $\begin{array}{l}\text { yes } \\
\text { No }\end{array}$ & $\begin{array}{l}35(8.9 \%) \\
360(91.1 \%)\end{array}$ \\
\hline Admission to ICU and need mechanical ventilation & & $15(3.8 \%)$ \\
\hline Admission to ICU and no need mechanical ventilation & & $20(5.1 \%)$ \\
\hline \multicolumn{3}{|l|}{ Outcome } \\
\hline Discharged home & & $386(97.7 \%)$ \\
\hline Died & & $9(2.3 \%)$ \\
\hline
\end{tabular}


Table 3. Epidemiologic characteristics association with hospital course and Outcome.

\begin{tabular}{|c|c|c|c|c|c|c|c|c|}
\hline \multirow[b]{2}{*}{ Attributes } & \multirow[b]{2}{*}{ Categories } & \multicolumn{4}{|c|}{ Hospital Course } & \multicolumn{3}{|l|}{ Outcome } \\
\hline & & $\begin{array}{l}\text { No ICU \& } \\
\text { No vent. }\end{array}$ & $\begin{array}{l}\text { Yes ICU \& } \\
\text { No vent. }\end{array}$ & $\begin{array}{l}\text { Yes ICU \& } \\
\text { Yes vent. }\end{array}$ & $\begin{array}{l}\text { Chi-square } \\
\text { P-value } \\
\end{array}$ & Discharged & $\begin{array}{l}\text { Passed } \\
\text { away }\end{array}$ & $\begin{array}{l}\text { Chi-square } \\
\text { P-value } \\
\end{array}$ \\
\hline \multirow{4}{*}{ Age Group } & $<18$ & $9(100 \%)$ & $0(0 \%)$ & $0(0 \%)$ & \multirow{4}{*}{$0.006^{* *}$} & $9(100 \%)$ & $0(0 \%)$ & \multirow{4}{*}{$0.013^{*}$} \\
\hline & $18-45$ & $192(94.1 \%)$ & $12(5.9 \%)$ & $0(0 \%)$ & & $204(100 \%)$ & $0(0 \%)$ & \\
\hline & $46-65$ & $125(86.2 \%)$ & $8(5.5 \%)$ & $12(8.3 \%)$ & & $138(95.2 \%)$ & $7(4.8 \%)$ & \\
\hline & $>65$ & $33(89.2 \%)$ & $2(5.4 \%)$ & $2(5.4 \%)$ & & $35(94.6 \%)$ & $2(5.4 \%)$ & \\
\hline Gender & Male & $250(89.9 \%)$ & $17(6.1 \%)$ & $11(4 \%)$ & $0.592^{\mathrm{NS}}$ & $272(97.8 \%)$ & $6(2.2 \%)$ & $0.728^{\mathrm{NS}}$ \\
\hline \multirow{3}{*}{ Ethnicity } & Caucasians & $15(50 \%)$ & $11(36.7 \%)$ & $4(13.3 \%)$ & \multirow{3}{*}{$0.260^{\mathrm{NS}}$} & $29(96.7 \%)$ & $1(3.3 \%)$ & \multirow{3}{*}{0.135} \\
\hline & Asian & $30(18 \%)$ & $20(12 \%)$ & $5(3 \%)$ & & $162(98 \%)$ & $3(2 \%)$ & \\
\hline & African & $126(63 \%)$ & $42(21 \%)$ & $33(16 \%)$ & & $196(99 \%)$ & $4(1 \%)$ & \\
\hline \multirow{2}{*}{ Nationality } & Saudi Arabian & $64(92.8 \%)$ & $3(4.3 \%)$ & $2(2.9 \%)$ & \multirow{2}{*}{$0.754^{\mathrm{NS}}$} & $68(98.6 \%)$ & $1(1.4 \%)$ & \multirow{2}{*}{$0.769^{\mathrm{NS}}$} \\
\hline & All others & $93(89.4 \%)$ & $7(6.7 \%)$ & $4(3.4 \%)$ & & $103(99.0 \%)$ & $1(1.0 \%)$ & \\
\hline \multirow{3}{*}{$\mathrm{BMI}^{\#}$} & $<24$ & $34(100 \%)$ & $0(0 \%)$ & $0(0 \%)$ & \multirow{3}{*}{$0.249^{\mathrm{NS}}$} & $34(100 \%)$ & $0(0 \%)$ & \multirow{3}{*}{$0.043^{*}$} \\
\hline & $24-30$ & $101(87.8 \%)$ & $10(8.7 \%)$ & $4(3.5 \%)$ & & $111(96.5 \%)$ & $4(3.5 \%)$ & \\
\hline & $>40$ & $10(76.9 \%)$ & $2(15.4 \%)$ & $1(7.7 \%)$ & & $12(92.3 \%)$ & $1(7.7 \%)$ & \\
\hline \multirow{2}{*}{ Smoking } & Yes & $75(96.2 \%)$ & $2(2.6 \%)$ & $1(1.3 \%)$ & $011^{N S}$ & $77(98.7 \%)$ & $1(1.3 \%)$ & $0014^{*}$ \\
\hline & No & $199(91.3 \%)$ & $14(6.4 \%)$ & $5(2.3 \%)$ & 0.41 & $216(99.1 \%)$ & $2(0.9 \%)$ & 0.014 \\
\hline $\mathrm{BCG}^{\# \#}$ Vaccine & Yes & $199(90.5 \%)$ & $14(6.4 \%)$ & $7(3.2 \%)$ & $0236^{\mathrm{NS}}$ & $216(98.2 \%)$ & $4(1.8 \%)$ & $0196^{\mathrm{NS}}$ \\
\hline BCG vaccine & No & $52(98.1 \%)$ & $1(1.9 \%)$ & $0(0 \%)$ & 0.236 & $53(100 \%)$ & $0(0 \%)$ & 0.196 \\
\hline & $\mathrm{A}+$ & $58(87.9 \%)$ & $163(7.6 \%)$ & $3(4.5 \%)$ & & $64(97 \%)$ & $2(3 \%)$ & \\
\hline & A- & $3(75 \%)$ & $5(25 \%)$ & $0(0 \%)$ & & $4(100 \%)$ & $0(0 \%)$ & \\
\hline & $\mathrm{B}+$ & $43(82.7 \%)$ & $5(9.6 \%)$ & $4(7.7 \%)$ & & $49(94.2 \%)$ & $3(5.8 \%)$ & \\
\hline Blood Group & B- & $2(100 \%)$ & $1(0 \%)$ & $0(0 \%)$ & $0.000^{* *}$ & $2(100 \%)$ & $0(0 \%)$ & $0.758^{\mathrm{NS}}$ \\
\hline & $\mathrm{AB}+$ & $11(91.7 \%)$ & $1(0 \%)$ & $00(\%)$ & & $12(100 \%)$ & $0(0 \%)$ & \\
\hline & $\mathrm{O}+$ & $72(92.3 \%)$ & $5(6.4 \%)$ & $1(1.3 \%)$ & & $77(98.7 \%)$ & $1(1.3 \%)$ & \\
\hline & O- & $7(100 \%)$ & $00(\%)$ & $0(0 \%)$ & & $7(100 \%)$ & $0(0 \%)$ & \\
\hline & None & $216(92.7 \%)$ & $12(5.2 \%)$ & $5(2.1 \%)$ & & $230(98.7 \%)$ & $3(1.3 \%)$ & \\
\hline & Cardiac Disease & $17(85 \%)$ & $2(10 \%)$ & $1(5 \%)$ & & $19(95 \%)$ & $1(5 \%)$ & \\
\hline & Diabetes mellitus & $104(\%)$ & $22(\%)$ & $14(\%)$ & & $113(95.8 \%)$ & $5(4.2 \%)$ & \\
\hline & Hypertension & $87(88.8 \%)$ & $7(7.1 \%)$ & $4(4.1 \%)$ & & $95(96.9 \%)$ & $3(3.1 \%)$ & \\
\hline co-moroialties & $\mathrm{COPD}^{\# \# \#}$ & $1(\%)$ & $0(0 \%)$ & $0(0 \%)$ & 0.000 & $1(100 \%)$ & $0(\%)$ & 0.43 \\
\hline & Interstitial Lung Disease & $1(100 \%)$ & $0(0 \%)$ & $0(0 \%)$ & & $1(100 \%)$ & $0(0 \%)$ & \\
\hline & Systematic Lupus eryhthematosis & $1(100 \%)$ & $0(0 \%)$ & $0(0 \%)$ & & $1(100 \%)$ & $0(0 \%)$ & \\
\hline & Rheumatoid Arthritis & $2(\%)$ & $0(0 \%)$ & $0(0 \%)$ & & $2(100 \%)$ & $0(0 \%)$ & \\
\hline & Pregnancy & $6(\%)$ & $0(0 \%)$ & $0(0 \%)$ & & $6(100 \%)$ & $0(0 \%)$ & \\
\hline & Liver Transplant & $1(\%)$ & $0(0 \%)$ & $0(0 \%)$ & & $1(100 \%)$ & $0(0 \%)$ & \\
\hline & Hydroxychloroquine & $137(85.1 \%)$ & $15(9.3 \%)$ & $9(\%)$ & & $156(96.9 \%)$ & $5(3.1 \%)$ & \\
\hline & Therapeutic anticoagulation & $138(81.2 \%)$ & $19(11.2 \%)$ & $13(7.6 \%)$ & & $161(94.7 \%)$ & $9(5.3 \%)$ & \\
\hline Medications & Steroids & $78(79.6 \%)$ & $11(11.2 \%)$ & $9(9.2 \%)$ & $0.000^{* *}$ & $91(92.9 \%)$ & $7(7.1 \%)$ & $0.000^{* *}$ \\
\hline & Tocilizumab & $57(74 \%)$ & $10(13 \%)$ & $10(13 \%)$ & & $57(74 \%)$ & $10(13 \%)$ & \\
\hline & Azithromycin & $113(87.6 \%)$ & $11(8.5 \%)$ & $5(3.9 \%)$ & & $113(87.6 \%)$ & $11(8.5 \%)$ & \\
\hline Symntoms & Symptoms & $301(89.3 \%)$ & $22(6.5 \%)$ & $14(4.2 \%)$ & $0033 *$ & $328(97.3 \%)$ & $9(2.7 \%)$ & $0208^{\mathrm{NS}}$ \\
\hline symptoms & No symptoms & $58(100 \%)$ & $0(0 \%)$ & $0(0 \%)$ & $0.033^{*}$ & $58(100 \%)$ & $0(0 \%)$ & \\
\hline $\mathrm{HRCT}^{\# \# \#}$ & Normal & $170(96.6 \%)$ & $5(2.8 \%)$ & $1(0.6 \%)$ & $0001 * *$ & $175(99.4 \%)$ & $1(0.6 \%)$ & $0030 *$ \\
\hline chest & Abnormal & $185(86 \%)$ & $17(7.9 \%)$ & $13(6 \%)$ & $0.001 \cdots$ & $207(96.3 \%)$ & $8(3.7 \%)$ & $0.039^{\circ}$ \\
\hline
\end{tabular}

\#BMI: body mass index.

\#\#BCG: Bacille Calmette Guerin.

\#\#\# COPD: chronic obstructive pulmonary disease.

\#\#\#\# HRCT: high resolution Ccomputed tomography.

Table 4. Epidemiologic characteristics association with findings on chest imaging.

\begin{tabular}{|c|c|c|c|c|c|c|c|}
\hline \multirow[b]{2}{*}{ Attributes } & \multirow[b]{2}{*}{ Categories } & \multicolumn{3}{|l|}{ CT Findings } & \multicolumn{3}{|l|}{ CxR Findings } \\
\hline & & Normal & Abnormal & $\begin{array}{l}\text { Chi-square } \\
\text { P-value }\end{array}$ & Normal & Abnormal & $\begin{array}{l}\text { Chi-square } \\
\text { P-value }\end{array}$ \\
\hline \multirow{4}{*}{ Age Group } & $<18$ & $9(100 \%)$ & $0(0 \%)$ & \multirow{4}{*}{$0.000^{* *}$} & $9(100 \%)$ & $0(0 \%)$ & \multirow{4}{*}{$0.000^{* *}$} \\
\hline & $18-45$ & $120(59.7 \%)$ & $81(40.3 \%)$ & & $120(58.8 \%)$ & $84(41.2 \%)$ & \\
\hline & $46-65$ & $43(29.9 \%)$ & $101(70.1 \%)$ & & $43(29.7 \%)$ & $102(70.3 \%)$ & \\
\hline & $>65$ & $4(10.8 \%)$ & $33(89.2 \%)$ & & $4(10.8 \%)$ & $33(89.2 \%)$ & \\
\hline Gender & Male & $118(42.6 \%)$ & $159(57.4 \%)$ & $0.135^{\mathrm{NS}}$ & $118(42.4 \%)$ & $160(57.6 \%)$ & $0.193^{\mathrm{NS}}$ \\
\hline
\end{tabular}




\begin{tabular}{|c|c|c|c|c|c|c|c|}
\hline \multirow[b]{2}{*}{ Attributes } & \multirow[b]{2}{*}{ Categories } & \multicolumn{3}{|l|}{ CT Findings } & \multicolumn{3}{|l|}{ CxR Findings } \\
\hline & & Normal & Abnormal & $\begin{array}{l}\text { Chi-square } \\
\text { P-value }\end{array}$ & Normal & Abnormal & $\begin{array}{l}\text { Chi-square } \\
\text { P-value }\end{array}$ \\
\hline \multirow{3}{*}{ Ethnicity } & Caucasians & $15(50 \%)$ & $15(50 \%)$ & \multirow{3}{*}{$0.528^{\mathrm{NS}}$} & $15(50 \%)$ & $15(50 \%)$ & \multirow{3}{*}{0.562} \\
\hline & Asian & $69(41.8 \%)$ & $96(58.2 \%)$ & & $65(40.0 \%)$ & $100(60.6 \%)$ & \\
\hline & African & $92(46.9 \%)$ & $104(53.1 \%)$ & & $92(46.9 \%)$ & $108(54.0 \%)$ & \\
\hline \multirow{3}{*}{ Nationality } & Saudi Arabian & $42(61.8 \%)$ & $26(38.2 \%)$ & \multirow{2}{*}{$0.937^{\mathrm{NS}}$} & $42(60.9 \%)$ & $27(39.1 \%)$ & \multirow{2}{*}{$0.969^{\mathrm{NS}}$} \\
\hline & All others & $63(61.2 \%)$ & $40(38.8 \%)$ & & $63(60.6 \%)$ & $41(39.4 \%)$ & \\
\hline & $<24$ & $22(64.7 \%)$ & $12(35.3 \%)$ & \multirow{4}{*}{$0.023^{*}$} & $22(64.7 \%)$ & $12(35.3 \%)$ & \multirow{4}{*}{$0.043^{*}$} \\
\hline \multirow{3}{*}{$\mathrm{BMI}^{\#}$} & $24-30$ & $53(46.1 \%)$ & $62(53.9 \%)$ & & $53(46.1 \%)$ & $62(53.9 \%)$ & \\
\hline & $30-40$ & $49(65.3 \%)$ & $26(34.7 \%)$ & & $26(33.8 \%)$ & $51(66.2 \%)$ & \\
\hline & $>40$ & $3(23.1 \%)$ & $10(76.9 \%)$ & & $3(23.1 \%)$ & $10(76.9 \%)$ & \\
\hline \multirow{2}{*}{ Smoking } & Yes & $45(57.7 \%)$ & $33(42.3 \%)$ & \multirow{2}{*}{$0.001^{* *}$} & $45(57.7 \%)$ & $33(42.3 \%)$ & \multirow{2}{*}{$0.000^{* *}$} \\
\hline & No & $113(52.6 \%)$ & $102(47.4 \%)$ & & $102(46.8 \%)$ & $116(53.2 \%)$ & \\
\hline \multirow{2}{*}{$\mathrm{BCG}^{\# \#}$ Vaccine } & Yes & $114(52.5 \%)$ & $103(47.5 \%)$ & \multirow{2}{*}{$0.004^{* *}$} & $114(51.8 \%)$ & $106(48.2 \%)$ & \multirow{2}{*}{$0.005^{* *}$} \\
\hline & No & $20(37.7 \%)$ & $33(62.3 \%)$ & & $20(37.7 \%)$ & $33(62.3 \%)$ & \\
\hline \multirow{7}{*}{ Blood Group } & $\mathrm{A}+$ & $35(53.8 \%)$ & $30(46.2 \%)$ & & $35(53.0 \%)$ & $31(47.0 \%)$ & \multirow{7}{*}{$0.285^{\mathrm{NS}}$} \\
\hline & A- & $3(75.0 \%)$ & $1(75.0 \%)$ & & $3(75.0 \%)$ & $1(25.0 \%)$ & \\
\hline & $\mathrm{B}+$ & $19(36.5 \%)$ & $33(63.5 \%)$ & & $19(936.5 \%)$ & $33(63.5 \%)$ & \\
\hline & B- & $1(50.0 \%)$ & $1(50.0 \%)$ & $0.232^{\mathrm{NS}}$ & $1(50.0 \%)$ & $1(50.0 \%)$ & \\
\hline & $\mathrm{AB}+$ & $6(50.0 \%)$ & $6(50.0 \%)$ & & $6(50.0 \%)$ & $6(50.0 \%)$ & \\
\hline & $\mathrm{O}+$ & $37(49.3 \%)$ & $38(50.7 \%)$ & & $37(47.4 \%)$ & $41(52.6 \%)$ & \\
\hline & O- & $5(71.4 \%)$ & $2(28.6 \%)$ & & $5(71.4 \%)$ & $2(28.6 \%)$ & \\
\hline \multirow{12}{*}{ Co-morbidities } & None & $134(57.5 \%)$ & $99(42.5 \%)$ & \multirow{12}{*}{$0.001^{* *}$} & $134(57.5 \%)$ & $99(42.5 \%)$ & \multirow{12}{*}{$0.001^{* *}$} \\
\hline & Cardiac Disease & $2(10 \%)$ & $18(90.0 \%)$ & & $2(10 \%)$ & $18(90 \%)$ & \\
\hline & Diabetes mellitus & $27(23.1 \%)$ & $90(76.9 \%)$ & & $27(22.9 \%)$ & $91(77.1 \%)$ & \\
\hline & Hypertension & $23(23.5 \%)$ & $75(76.5 .1 \%)$ & & $23(23.5 \%)$ & $75(76.5 \%)$ & \\
\hline & Chronic kidney disease & $1(7.7 \%)$ & $12(92.3 \%)$ & & $1(7.7 \%)$ & $12(92.3 \%)$ & \\
\hline & Human immunodeficiency virus & $0(0.0 \%)$ & $1(100 \%)$ & & $1(33.3 \%)$ & $266.7(\%)$ & \\
\hline & $\mathrm{COPD}^{\# \# \#}$ & $0(0.0 \%)$ & $1(100 \%)$ & & $0(0.0 \%)$ & $1(100 \%)$ & \\
\hline & Interstitial Lung Disease & $0(0.0 \%)$ & $0(0.0 \%)$ & & $0(0.0 \%)$ & $0(0.0 \%)$ & \\
\hline & Systematic Lupus eryhthematosis & $1(100 \%)$ & $0(0 \%)$ & & $1(100 \%)$ & $0(0 \%)$ & \\
\hline & Rheumatoid Arthritis & $1(50.0 \%)$ & $1(50.0 \%)$ & & $1(50.0 \%)$ & $1(50.0 \%)$ & \\
\hline & Pregnancy & $1(33.3 \%)$ & $2(66.7 \%)$ & & $1(16.7 \%)$ & $5(83.3 \%)$ & \\
\hline & Liver Transplant & $0(0.0 \%)$ & $1(100.0 \%)$ & & $0(0.0 \%)$ & $1(100 \%)$ & \\
\hline
\end{tabular}

\#BMI: body mass index.

\#\#BCG: Bacille Calmette Guerin.

\#\#\# COPD: chronic obstructive pulmonary disease.

\section{Discussion}

In this paper we reviewed the epidemiologic and clinical characteristics of 395 virologically confirmed Covid 19 patients. Males accounted for majority of patients in our population (Around two third were males vs one third females); this is unlike what has been reported in an earlier paper from Saudi Arabia where males accounted for $54.3 \%$ and females for $45.7 \%$ [10].

The most common clinical presentation in our population was pneumonia $(63.8 \%)$, followed by upper respiratory tract infection $(21.2 \%)$ and then asymptomatic state $(15 \%)$. Among the symptomatic patients, the most common symptom was fever $(74.2 \%)$ followed by cough $(65.6 \%)$, and shortness of breath $(40.3 \%) .15 .2 \%$ of our patients had diarrhea. The majority had fatigue, headache and myalgias. Fewer patients had anosmia, dysgeusia, sore throat and runny nose. Our findings are consistent with findings reported in some other published series $[7,11,12]$. We had some variations in our population: Only one of our patients reported sputum production, unlike some data reporting this finding in up to $53 \%$ of patients $[7,11]$. No ocular manifestations were reported in our population. This is unlike the $31 \%$ ocular manifestations reported in a Covid 19 paper by $\mathrm{Wu}$, et al [13]. Diarrhea was reported in $15 \%$ of of our population, similar to prevalence in the paper published from Saudi Arabia [10], but higher than the 2- 10\% reported in other series $[14,15]$. Variations could be due to the subjective nature of the data and to the period of analysis of patients. Other likely explanation for variation could be the different genetic makeup of our patient population from other populations; this is in view of published data on identification of a gene cluster on chromosome 3 affecting clinical presentation and risk factor for severe disease [16].

Around $15 \%$ of our patients were completely asymptomatic and were admitted to the hospital because hospitals in Saudi Arabia were mandated by ministry of health during first quarter of 2020 to admit any virologically confirmed patients regardless of symptoms. This practice was stopped later in April. Paper by Alsofyan et al from Saudi Arabia [10] showed that asymptomatic patients accounted for $9 \%$. This difference could be due to the period of data collection and analysis.

It is noteworthy that in our population around one quarter of asymptomatic patients had abnormal chest imaging mainly consisting of ground glass opacities and 42.7 percent had lymphocytopenia (lymphocytes less than 24\%), findings 
similar to data reported in earlier publications $[17,18]$. These findings in asymptomatic patients show that Covid 19 asymptomatic state is more of a silent active infection rather than a silent inactive carrier state. This active silent infection has significant asscoaition with good outcome as none of our asymptomatic patients needed ICU care and none died.

Mortality in our series was $2.3 \%$. Studies conducted in china showed mortality rate (reported death divided by reported cases) of $3.6 \%$ [19]. Difference in mortality rate could be accounted for by difference in the denominator since different countries had different Covid 19 testing strategies and solid comparisons between institutions cannot be made.

Increased risk of death was significantly associated in our population with advancing age, increasing BMI and history of smoking. Findings consistent with prior publications [20, 21]. Gender has been reported to be an independent risk factor for mortality [22], but not in our populations where Gender and ethnicity had no significant association with mortality and outcome. Genetic characteristics of different populations could explain the discrepancy.

The fact that we adopted different management protocols over the 4 months that spanned the study duration gave us opportunity to assess effect of different therapeutic interventions on patients' outcome. Our findings show significant association of good outcome with combination of hydroxychloroquine and azithromycin and negative association with Tocilizumab. This could be explained by the fact that Tocilizumab was given only to patients with desaturation and with respiratory distress. Considering that this is an observational study we cannot make significant associations or conclusions on efficacy of medications.

Universal BCG vaccination at birth is standard practice in Saudi Arabia, hence the high percentage of our patients that had positive history of vaccinations. Published reports suggested an association between prior BCG vaccination and severity of Covid 19 disease and outcome [23-25]. On April $12^{\text {th }}, 2020$ WHO release denied any strong proof to make such association and stated that 2 RCT's are underway to evaluate this point. In our population we found that BCG vaccination was significantly associated with less lower respiratory tract involvement (pneumonia). No significant association was shown with need for ICU care or with mortality. The observational nature of our study makes us unable to make strong conclusions.

Almost half of patients in our population denied any history of exposure to patients with Respiratory illnesses or history of contact with Covid 19 patients, underscoring the importance of transmission by asymptomatic carriers. Our findings are similar to reports from other centers. Transmission of SARS Co-v2 by asymptomatic patients was first reported by Bai et al from Wuhan [26] and then reported from other centers [27].

In addition to the challenge of managing infected patients, the Covid 19 epidemic posed the serious challenge on clinicians not to miss other etiologies of febrile / respiratory illnesses that have similar presentations. Amid this epidemic we needed to constantly remind our clinicians to consider etiologies and pathogens other than SARS Cov-2 in patients presenting with febrile illness or with abnormalities on chest imaging. This tendency to focus only on Covid 19 may deprive patients of therapeutic interventions that are warranted but missed due to the narrow differential diagnosis by clinicians. That is why we tried during analysis of our data to identify findings that not only confirm but also that help exclude diagnosis of Covid 19. We noted for example that Covid 19 patients in our population rarely dropped platelets to less than 100,000, rarely had CRP higher than 150, and rarely had ALT higher than 3 times upper normal. Such clues and findings in patients should prompt clinicians to investigate and try to rule out etiologies other than Covid 19.

\section{Conclusion}

Advancing age, increasing BMI and history of smoking were found to be significant risk factors for mortality in our population. History of BCG vaccination was significantly associated with less involvement of lower respiratory tract but had no significant association with final outcome of patients. Covid 19 in asymptomatic patients is more of a silent active infection rather than a silent inactive carrier state. In our population, findings at presentation that may suggest differential diagnoses other than Covid 19 in patients presenting with respiratpry illness include significant thrombocytopenia (platelets less than 100,000), CRP higher than 150 and ALT higher than 3 times upon normal.

\section{References}

[1] World Health Organization. https://www.who.int/dg/speeches/detail/who-director-generals-opening-remarks-at-the-media-briefing-on-covid-19-11march-2020, 2020. March [Accessed 4 April 2020].

[2] $\mathrm{MOH}$

News. https://www.moh.gov.sa/en/Ministry/MediaCenter/News/Page s/News-2020-03-02-002.aspx, 2020. March [Accessed 5 April 2020].

[3] Mizumoto K, Kagaya K, Zarebski A, Chowell G. Estimating the asymp-tomatic proportion of coronavirus disease 2019 (COVID-19) cases on boardthe Diamond Princess cruise ship, Yokohama, Japan, 2020. Eurosurveillance 2020; 25 (10), http://dx.doi.org/10.2807/1560-7917.ES.2020.25.10.2000180.

[4] Guan W, Ni Z, Hu Y, Liang W, Ou C, He J, et al. Clinical characteristics of Corona-virus Disease 2019 in China. New Engl J Med 2020, http://dx.doi.org/10.1056/NEJMoa2002032.

[5] Jiang F, Deng L, Zhang L, Cai Y, Cheung C, Xia Z. Review of the clinical character-istics of Coronavirus Disease 2019 (COVID-19). J Gen Intern Med 2020, http://dx.doi.org/10.1007/s11606-020-05762-w.

[6] Pan L, Mu M, Yang P, Sun Y, Wang R, Yan J, et al. Clinical characteristics of COVID-19 patients with digestive symptoms in Hubei, China. Am J Gastroen-terol 2020: 1, http://dx.doi.org/10.14309/ajg.0000000000000620. 
[7] Huang C, Wang Y, Li X, Ren L, Zhao J, Hu Y, et al. Clinical features of patients infected with 2019 novel coronavirus in Wuhan, China. Lancet. 2020; 395 (10223): 497-506.

[8] Wang D, Hu B, Hu C, Zhu F, Liu X, Zhang J, et al. Clinical characteristics of 138 hospitalized patients with 2019 Novel Coronavirus-Infected Pneumoniain Wuhan, China. J Am Med Assoc2020; $323 \quad$ (11): 1061 , http://dx.doi.org/10.1001/jama.2020.1585.

[9] Shi H, Han X, Jiang N, Cao Y, Alwalid O, Gu J, et al. Radiological findingsfrom 81 patients with COVID-19 pneumonia in Wuhan, China: a descriptivestudy. Lancet Infect Dis 2020 ; 20 (4): 425-34, http://dx.doi.org/10.1016/S14733099(20)30086-4.

[10] Alsofyan Y, Althunayyan S, Khan A, Hakawi A, Assiri A (2020) Clinical characteristics of Covid-19 in Saudi Arabia: a national retrospective study. $\mathrm{J}$ Infect and public health. https://doi.org/10.1016/j.jiph.2020.05.026.

[11] Liu KC, Xu P, Lv WF, Qiu XH, Yao JL, Gu JF, et al. CT manifestations of coronavirus disease-2019: a retrospective analysis of 73 cases by disease severity. Eur J Radiol. 2020 May; 126: 108941. doi: 10.1016/j.ejrad.2020.108941.

[12] Linton NM, Kobayashi T, Yang Y, Hayashi K, Akhmetzhanov AR, Jung SM, et al. Incubation period and other epidemiological characteristics of 2019 novel coronavirus infections with right truncation: a statistical analysis of publicly available case data. J Clin Med. 2020; 9 (2): 538. doi: $10.3390 / \mathrm{jcm} 9020538$.

[13] Wu P, Duan F, Luo C, Liu Q, Qu X, Liang L, et al. Characteristics of ocular findings of patients with coronavirus disease 2019 (COVID-19) in Hubei Province, China. JAMA Ophthalmol. 2020; e201291. doi: 10.1001/jamaophthalmol.2020.1291.

[14] Chen N, Zhou M, Dong X, et al. Epidemiological and clinical characteristics of 99 cases of 2019 novel coronavirus pneumonia in Wuhan, China: a descriptive study. Lancet 2020; 395: 507-13. doi: 10.1016/S0140-6736(20)30211-7.

[15] Wang D, Hu B, Hu C, et al. Clinical characteristics of 138 hospitalized patients with 2019 novel coronavirus-infected pneumonia in Wuhan, China. JAMA 2020. doi: 10.1001/jama.2020.1585.

[16] Ellinghaus D, Degenhardt F, Bujanda L, Buti M, Albillos A, et al (2020). Genomewide Association Study of Severe Covid-19 with Respiratory Failure, NEJM 2020; DOI: 10.1056/NEJMoa2020283.
[17] Yu C, Zhou M, Liu Y, Guo T, Ou C, et al (2020). Characteristis of asymptomatic Covid 19 infection and progression: A multicenter, retrospective study. Virulence $2020 ; 11$, issue 1 .

[18] Long Q, Tang X, Shi Q, Li Q, Deng H, et al (2020). Clinical and immunological asessment of asymotomatic SARS-Cov-2 infections. Nature Medicine 2020; 26: 1200-1204.

[19] Baud D, Qi X, Nielsen-Saines K, Musso D, Pomar L, Favre G. Real estimates of mortality following COVID-19 infection. Lancet Infect Dis. 2020 Mar 12;. doi: 10.1016/S14733099(20)30195-X.

[20] Zhou F, Yu T, Du R, Fan G, Liu Y, Liu Z, et al. Clinical course and risk factors for mortality of adult inpatients with COVID19 in Wuhan, China: a retrospective cohort study. Lancet 2020; 395 (10229): 1054-62.

[21] Wu C, Chen X, Cai Y, Xia J, Zhou X, Xu S, et al. Risk factors associated with acute respiratory distress syndrome and death in patients with coronavirus disease 2019 pneumonia in Wuhan, China. March 2020. JAMA Intern Med. 2020 Mar 13; e200994. doi: 10.1001/jamainternmed.2020.0994.

[22] Wang D, Yin Y, Hu C, Liu X, Zhang X, et al. Clinical course and outcome of 107 patients infected with Novel coronavirus, SARS Co-v- 2, discharge dfrom 2 hopsitals in Wuhan. Critical care 2020; 24: 188.

[23] Miller A, Reandelar MJ, Fasciglione K, Roumenova V, Li Yan, Otazu GH. Correlation between universal BCG vaccination policy and reduced morbidity and mortality for COVID- 19: an epidemiological study. 2020 medRxiv doi: 10.1101/2020.03.24.20042937.

[24] Hegarty PK, Kamat AM, Zafirakis H, Dinardo A. BCG vaccination may be protective against Covid-19. 2020. doi: 10.13140/RG.2.2.35948.10880.

[25] Dayal D, Gupta S (2020). Connecting BCG vaccination and Covid 19: additional data. medRxiv preprint doi: https://doi.org/10.1101/2020.04.07.20053272.

[26] Bai Y, Yao L, Wei T, Jin D, et al (2020). Presumed asymptomatic carrier transmission of Covid 19 JAMA. 2020 Apr 14; 323 (14): 1406-1407.

[27] Arnos MM, Hatfield KM, Reddy SC, Kimball A, James A, et al (2020). Presymptomatic SARS-Cov-2 infections and transmission in a skilled nursing facility. N Engl J Med 2020; 382: 2081-2090. 